\title{
METAL MATRIX COMPOSITE SOLAR CELL METALLIZATION
}

\author{
David M. Wilt( ${ }^{(1)}$, Geoffrey Bradshaw ${ }^{(1)}$, Lt. Nathan Gap ${ }^{(1)}$, Omar K. Abudayyeh ${ }^{(2)}$, Cayla Nelson ${ }^{(2)}$, Sang Han ${ }^{(2)}$, \\ Nathanael Cox ${ }^{(3)}$, Aaron Rape ${ }^{(3)}$, Brian Landi ${ }^{(3)}$ and Steve Whipple ${ }^{(4)}$ \\ (I) Space Vehicles Directorate, Air Force Research Laboratory \\ 3550 Aberdeen Ave SE, Kirtland Air Force Base, NM 87111,USA, AFRL.VSSVOrgMailbox@us.af.mil \\ ${ }^{(2)}$ The University of New Mexico, Albuquerque, NM USA \\ ${ }^{(3)}$ Rochester Institute of Technology, Rochester, NY, USA \\ (4) SolAero Technologies Corp, Albuquerque, NM, USA
}

\begin{abstract}
Advanced solar cells are moving to ever thinner formats in order to save mass and in some cases improve performance. As cells are thinned, the possibility that they may fracture or cleave due to mechanical stresses is increased. Fractures of the cell can degrade the overall device performance if the fracture propagates through the contact metallization, which frequently occurs. To address this problem, a novel semiconductor metallization system based on multi-walled carbon nanotube (CNT) reinforcement, termed metal matrix composite (MMC) metallization is under investigation. Electro-mechanical characterization of MMC films demonstrate their ability to provide electrical conductivity over $>40$ micron wide cracks in the underlying semiconductor, with the carbon nanotubes bridging the gap. In addition, these materials show a "self-healing" behaviour, electrically reconnecting at $\sim 30$ microns when strained past failure. Triple junction (TJ) space cells with MMC metallization demonstrated no loss in $\mathrm{J}_{\mathrm{sc}}$ after intentional fracture, whereas $\mathrm{TJ}$ cells with conventional metallization suffer up to $50 \% \mathrm{~J}_{\mathrm{sc}}$ loss.
\end{abstract}

\section{INTRODUCTION}

Advanced crystalline solar cells used on-earth and inspace are rapidly moving towards thinner devices. For terrestrial applications, the emphasis on thinner devices is driven primarily to reduce material costs, although there can be device performance benefits. In space, thinner triple junction cells are becoming more common due to their mass savings and inverted metamorphic multijunction (IMM) solar cells, consisting of 10 to 15 microns of epitaxy, are nearing market entry. Despite the advantages of thinner crystalline solar cells, they could be more likely to fracture and cleave in response to mechanical stress. Frequently, fractures that remain within the semiconductor layers do not severely impact the overall device performance. Unfortunately, high stresses at the crack tips generally lead to fracturing of the metallization and subsequent loss of electrical performance. Recently, Kajari-Schröderet al. [1] have characterized microcracks in crystalline-silicon-based photovoltaic cells, using electroluminescence after artificial aging and snow damage (Fig. 1). These microcracks can electrically disconnect areas of the cells and lead to substantial power loss [2]. To mitigate the power loss and increase the lifetime of solar cells, we have developed and investigated silver ( $\mathrm{Ag})$ metal films imbedded with single and multi-walled carbon nanotubes (CNTs), also known as metal matrix composites (MMCs), for the reinforced mechanical strength against stress-induced cracks [3, 4, 5]. Numerous techniques exist today to deposit bulk MMCs, including powder metallurgy, melting and solidification, and electrochemical routes [6].

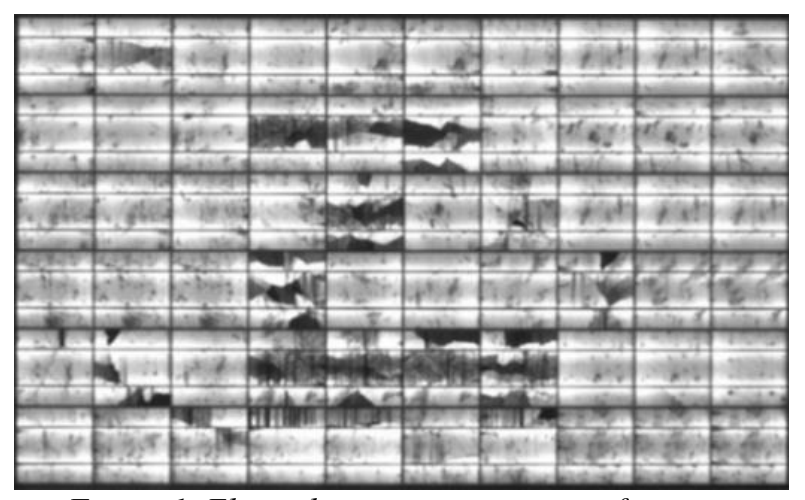

Figure 1. Electroluminescence image of a microcracked PV module. Dark regions are electrically inactive areas [1].

In this investigation, MMC films have been deposited by either metal electroplating combined with CNT spray coating or by metal evaporation combined with CNT film transfer, although some preliminary investigation of screen printing MMC films has also been conducted [7]. Both single wall and multi-wall CNT materials have been examined and the MMC structure has primarily consisted of a layer-by-layer design $(\sim 2 \mu \mathrm{m}$ Ag base layer / CNT film / 2 $\mu \mathrm{m} \mathrm{Ag} \mathrm{overcoat).} \mathrm{A} \mathrm{range} \mathrm{of} \mathrm{CNT}$ film loadings have been examined to assess the impact on MMC gapping capability (distance that the MMC films are able to electrically bridge). 
MMC electro-mechanical crack bridging capability was examined using a specifically designed test apparatus, affectionately called the RACK (Resistance Across Cleaves and cracKs). For this testing, MMC grid lines were formed on a semi-insulating indium phosphide (InP) substrate. InP was chosen as it is electrically insulating and more importantly, particularly easy to cleave. The sample is then bonded across two circuit boards and then mounted to a micro-positioning stage. The grid lines are then connected to the data acquisition system allowing $\sim 30 \mathrm{~mA}$ of current to be passed through each gridline and their resistance to be measured. A single cleave is then formed in the sample, perpendicular to the grid lines. The conductivity of the grid lines is then measured as the circuit boards are pulled apart in micron increments (and brought back together). The fracture spacing (gap width) is measured via optical microscope. Figure 2 shows a drawing of the overall RACK configuration and a photo of a sample under test.
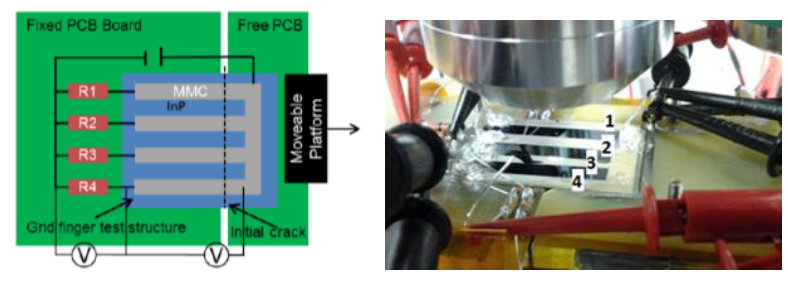

Figure 2. (l) drawing of RACK configuration (note: two voltage measurements were actually made for each of the four (4) gridlines), (r) photo of sample during RACK testing.

\section{MMC Electro-Mechanical Behavior}

Figure 3 shows the RACK data from pure $\mathrm{Ag}$ metallization and a single-walled CNT MMC (SWMMC) sample [5]. All of the Ag samples and many of the SW-MMC samples failed electrically at the initial crack. This is partly due to the $2 \mu \mathrm{m}$ to $>6 \mu \mathrm{m}$ initial crack that formed when the sample was cleaved. The shaded region indicates the initial crack length. The Agonly electrode fails electrically upon cracking (crack width $\sim 3.7 \mu \mathrm{m}$ ), while the SW-MMC maintains electrical connection after initial fracture (crack width $\sim 2.8 \mu \mathrm{m}$ ), and fails electrically after an additional extension of $2.7 \mu \mathrm{m}$, with a total gap width of $\sim 5.5 \mu \mathrm{m}$. This is representative of the successful SW-MMC samples, which generally failed below $6 \mu \mathrm{m}$.

Figure 4 shows the maximum and average gaps before the loss of electrical connection for multi-walled CNT MMC samples as a function of CNT loading [3]. The pull tests reveal that the electrical connection is maintained across larger gaps with higher CNT surface coverage, reaching a maximum of $42-\mu \mathrm{m}$-wide gap. As the CNT surface coverage increases from $35 \%$ to $96 \%$, we observe a monotonic increase in the average gap. Electroplated Ag lines without CNTs are also analyzed as controls, using RACK test. Unlike MMC films, the $\mathrm{Ag}$ gridlines did not withstand the initial crack generation ( $\sim 4-\mu \mathrm{m}$-wide), and the electrical connection is immediately and irrecoverably lost.

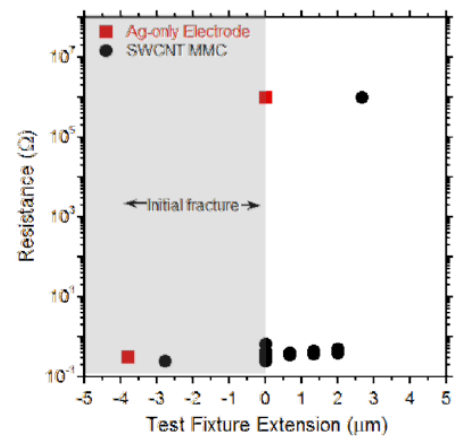

Figure 3 - Electrical resistance versus gap for a Agonly electrode compared to a single walled MMC electrode. The shaded region indicates the initial crack length.

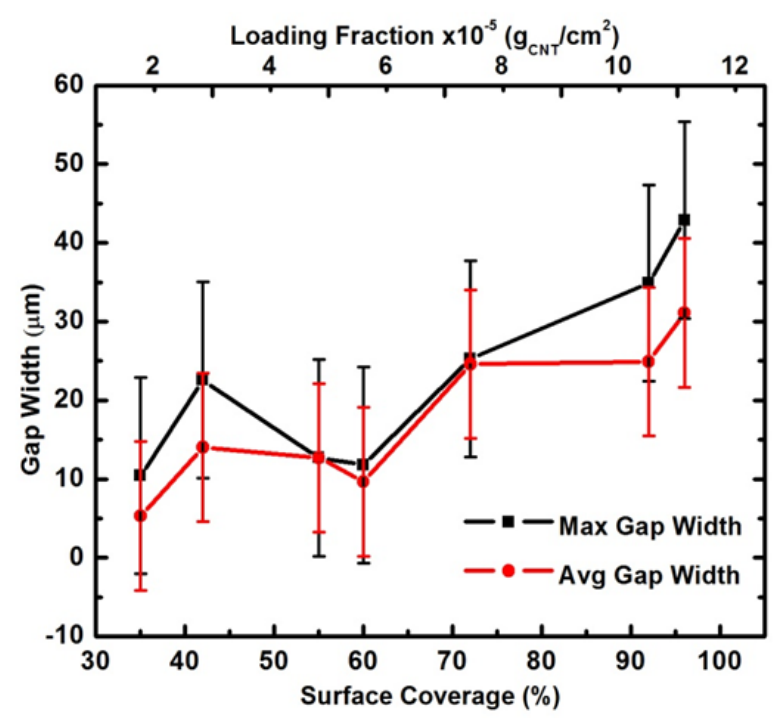

Figure 4 - Maximum and average gap widths achieved before lost connection as a function of CNT surface coverage and loading fraction.

SEM micrograph images of fractured MMC samples show how the multi-walled MMC metallization is able to maintain electrical connection across significant gaps ( $>40$ microns). Figure 5 shows the multi-walled CNT bridging a 9 micron wide fracture. The CNTs are anchored in the Ag matrix, indicating a good adhesion between the functionalized CNTs and the Ag matrix. Once fractured, the total current $(30 \mathrm{~mA})$ is conducted through only a limited number of CNTs at the fracture point, as shown in Fig. 5. We have not determined the maximum current density that MMCs can tolerate after 
fracture, but have pushed them to $150 \mathrm{~mA}\left(2500 \mathrm{~A} / \mathrm{cm}^{2}\right.$ equivalent) without conductivity loss.
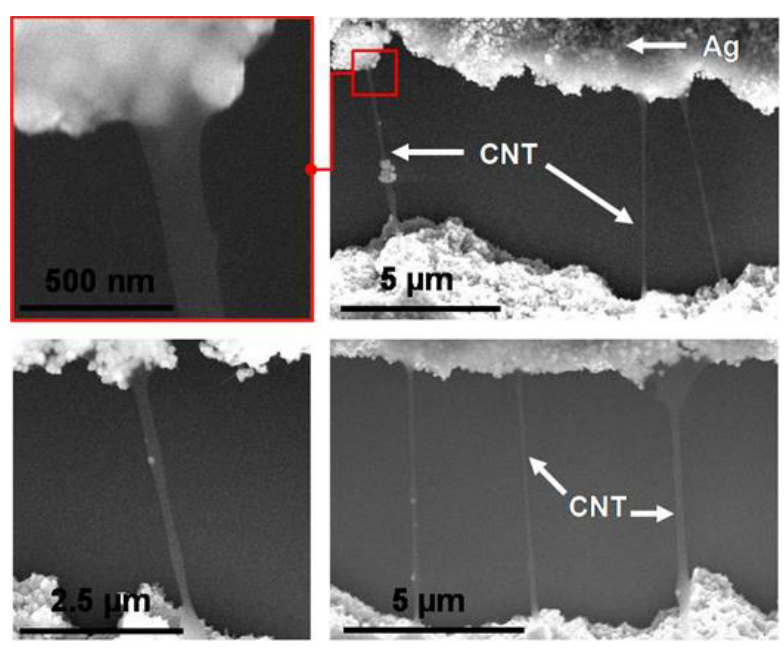

Figure 5 - SEM images of CNTs anchored in Ag layers clearly showing CNTs bridging cracks up to 9- $\mu$ m-wide.

As a test, MMC samples were pulled apart until they lost electrical contact and then were slowly brought back together while checking for electrical connection. Surprisingly, the MMC samples re-established electrical connection well before the sample edges were brought back into contact. Figure 6 shows the log of resistance as a function of physical gap for samples with different MWCNT loading as the gap is closed. The sample with the highest CNT loading showed electrical reconnection at $>40$ microns with the lower loaded samples reconnecting at $<10$ microns. It is believed that strong electric fields at the ends of the dangling CNT fibers is likely responsible for assisting them in reconnecting before the surfaces are brought in contact.

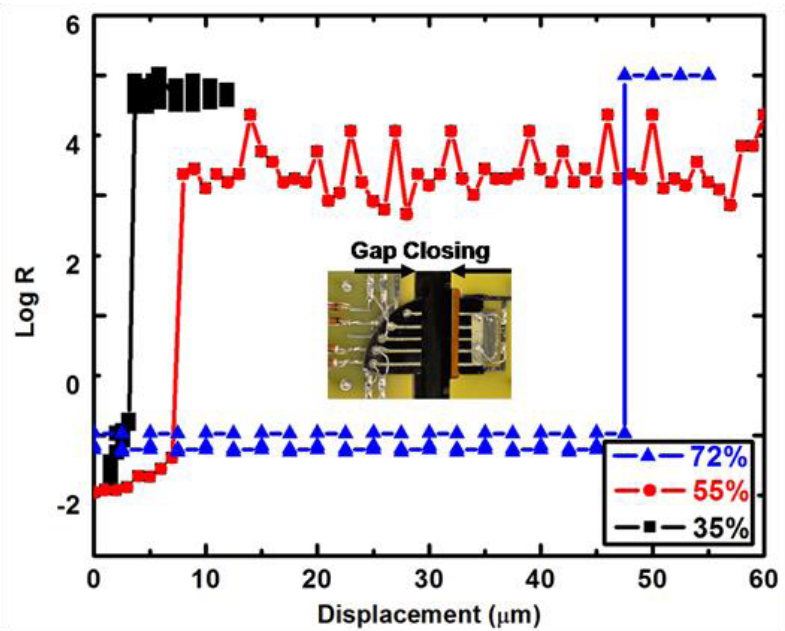

Figure 6 - Log plot of resistance vs. gap across fractured MMC gridlines with three different MWCNT surface coverages.
In order to test to see if this "self-healing" behaviour was repeatable, one sample was tested numerous times (pulled to electrical isolation, brought back together and then pulled apart again, $\sim 10 \mathrm{x}$ ). The data (figure 7) shows that for the second and third pulls, the maximum gap distance was reduced from $\sim 40$ microns to $\sim 25$ microns, but was stable at that point for all of the successive pulls.

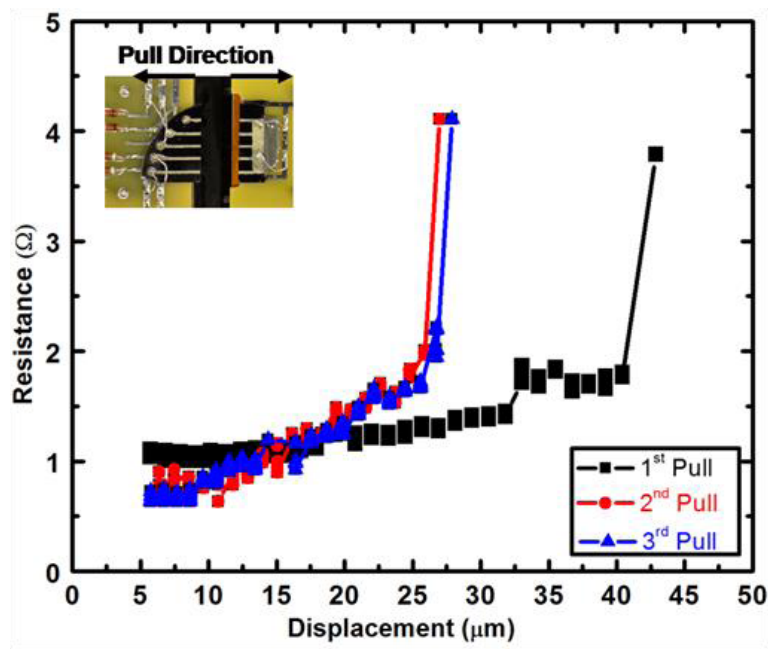

Figure 7 - Strain failure test of a MMC gridline $196 \%$ CNT surface coverage using drop cast method). The maximum gap, at which the electrical connection is lost, is reduced by $\sim 34 \%$ after the 2 nd pull test but remained constant after subsequent pull tests

\section{MMC Cell Integration}

Representative InGaP/GaAs/Ge space triple junction cells were fabricated, some with conventional evaporated metallization (controls) and some with only thin $(\sim 1000 \mathrm{~A})$ evaporated seed layers for the grid metallization. The cells with the seed layers had their metallization built up as either electroplated $\mathrm{Ag}$ or MMC consisting of electroplated $\mathrm{Ag}$ and spray deposited multi-wall CNT. I-V measurements of the devices demonstrated relatively comparable initial electrical performance ( $27 \%$ AM0 efficiency) for all of the devices.

The $2 \times 2 \mathrm{~cm}$ cells were then intentionally cracked by wrapping them around a $6 \mathrm{~cm}$ radius cylinder (back of the cell to the cylinder). Figure 8 shows the light I-V (LIV) measurements as well as the electroluminescence images of the control cells pre and post cracking. The top two cells have evaporated Ag metallization whereas the bottom two cells have electroplated Ag metallization. The EL and I-V data show that fracturing can sever the metallization, leading to a reduction in cell collecting area and frequently a significant ( $\sim 50 \%)$ reduction in $\mathrm{J}_{\mathrm{sc}}$. This data also suggests that there isn't a 
significant difference in the response of the cells to cracking based on the metal deposition process (evaporation vs. plating).
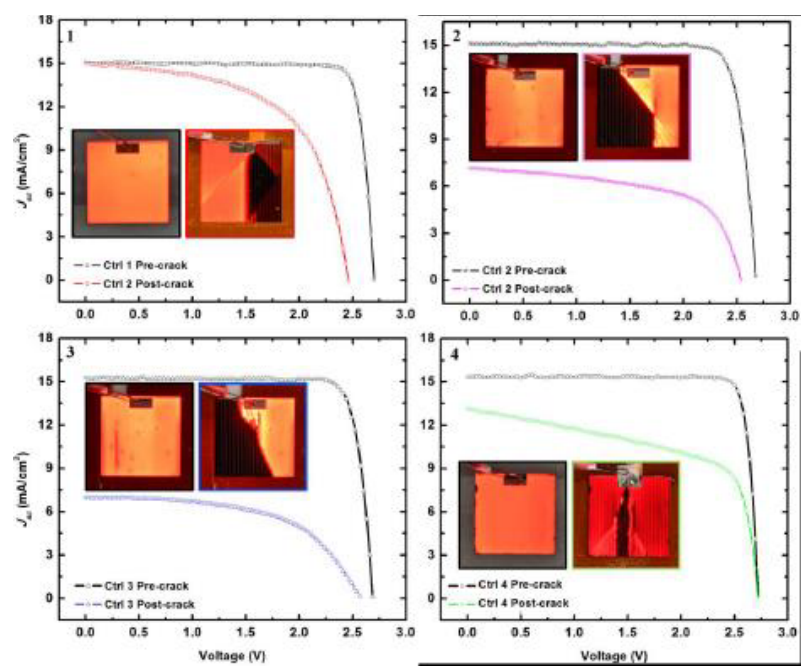

Figure 8-LIV characteristics and electroluminescence response of TJ control samples pre-and post-cracking.

Graphs (1) \& (2) represent control samples with electron beam evaporated Ag gridlines. Graphs (3) \& (4) represent control samples with electrochemically deposited Ag gridlines.
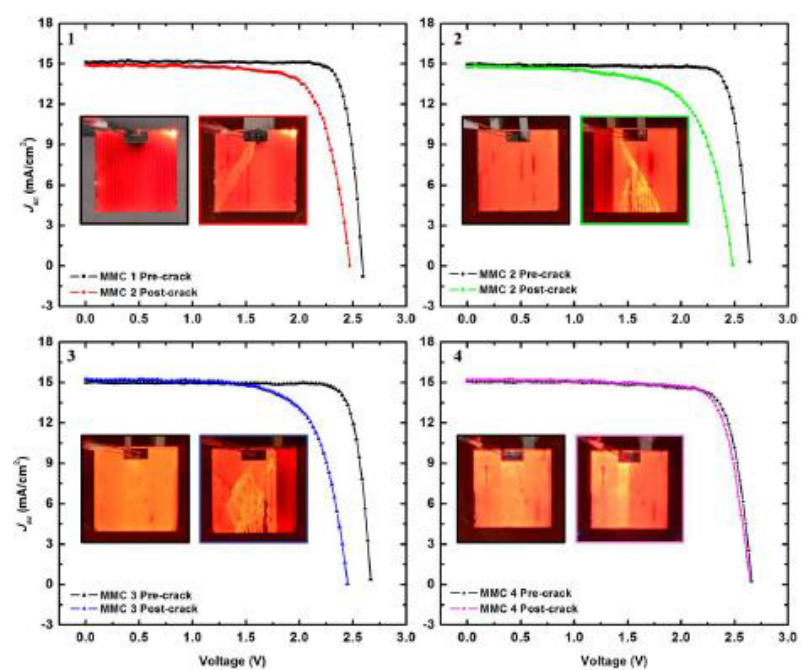

Figure 9-LIV characteristics and electroluminescence response of TJ cell with MMC gridlines.

Figure 9 shows the LIV and electroluminescence data for the cells with MMC metallization. The striking feature of this data set is the lack of $\mathrm{J}_{\mathrm{sc}}$ degradation due to cracking when MMC metallization is used. The cells do show variable $\mathrm{V}_{\mathrm{oc}}$ and $\mathrm{FF}$ degradation, but the current is essentially unchanged. This is particularly important as cells are connected in series, thus current loss in a single cell can limit the overall string. This data represents the first integration of MMC metallization with multijunction solar cells and further analysis and optimization is currently underway.

\section{CONCLUSIONS}

In this study, we have developed and characterized AgCNT metal matrix composite (MMC) films that maintain electrical conductivity upon mechanical fracture of the substrate. Our composite lines are capable of bridging cracks in the underlying semiconductor substrates $>40 \mu \mathrm{m}$. In addition to maintaining the electrical conductivity over the stressinduced cracks, the composite films can re-establish the electrical connection when the cracks close up. This MMC technology has been integrated with Ge based triple junction cells and has demonstrated a clear benefit in terms of $\mathrm{J}_{\mathrm{sc}}$ maintained following cell cracking. Additional work is underway to optimize and fully characterize this technology.

\section{REFERENCES}

1. S. Kajari-Schröder, Kunze I, U. Eitner, and M. Köntges, "Spatial and orientational distribution of cracks in crystalline photovoltaic modules generated by mechanical load tests," Solar Energy Mater Solar Cells, vol. 95, pp. 3054-3059, 2011.

2. M. Köntges, I. Kunze, S. Kajari-Schröder, X. Breitenmoser, and B. Bjrneklett, "The risk of power loss in crystalline silicon based photovoltaic modules due to microcracks," Solar Energy Mater Solar Cells, vol.95, pp. 1131-1137, 2011.

3. O. Abudayyeh, N. Gapp, C. Nelson, D. Wilt, and S. Han, "Silver-Carbon-Nanotube Metal Matrix Composites for Metal Contacts on Space Photovoltaic Cells," IEEE J. of Photovoltaics, 2015

4. O. Abudayyeh, D. Wilt, G. Bradshaw and S. Han, "Crack-Tolerant Silver-Carbon-Nanotubes Metal Matrix Composites as Photovoltaic Gridlines," IEEE J. of Photovoltaics, 2016 (in review)

5. N. Cox, M. Pham, N. Gapp, A. Rape, J. Rossi, D. Wilt, B. Landi, "Electrical Characterization of Carbon Nanotube Metal Matrix Composite Solar Cell Electrodes Under Mechanical Stress," Proc. $43^{\text {rd }}$ IEEE Photovoltaic Specialist Conference, 2016.

6. S. R. Bakshi, D. Lahiri, and A. Agarwal, "Carbon nanotube reinforced metal matrix composites - a review," International Materials Reviews, vol. 55, pp. 41-64, 2010.

7. A. Manoussakis, O. Abudayyeh, N. Gapp and D. Wilt, "Screen Printed Metal Matrix Composite Contacts for Crack Tolerant Solar Cells," Proc. 43rd IEEE Photovoltaic Specialist Conference, 2016. 